\title{
Creating Voluntary Payment Programs Effective Program Design and Ranchers' Willingness to Conserve Florida Panther Habitat
}

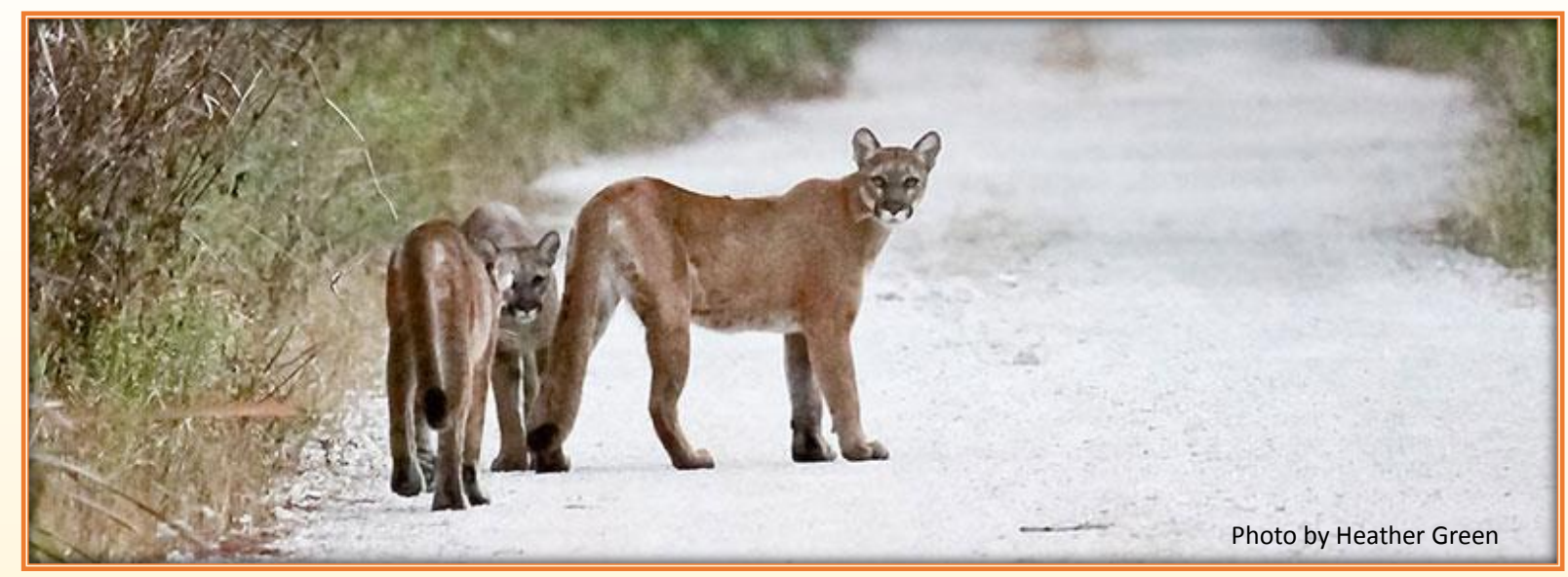

Elizabeth F. Pienaar, Melissa M. Kreye, José R. Soto, Damian C. Adams University of Florida

Paper forthcoming in Land Economics

Presented to: IWRS, Namibia

September 2016 


\section{Florida Panther}

- 1967: listed as endangered

- Population estimate: 100 - 180

- Recovery of the Florida panther:

- 3 populations ( $\geq 240$ adults $\&$ subadults)

- Maintain for $\geq 12$ years

- Secure and protect habitat of sufficient quality, quantity and spatial configuration to support panther in the long run

- Natural dispersal of panthers and gene flow

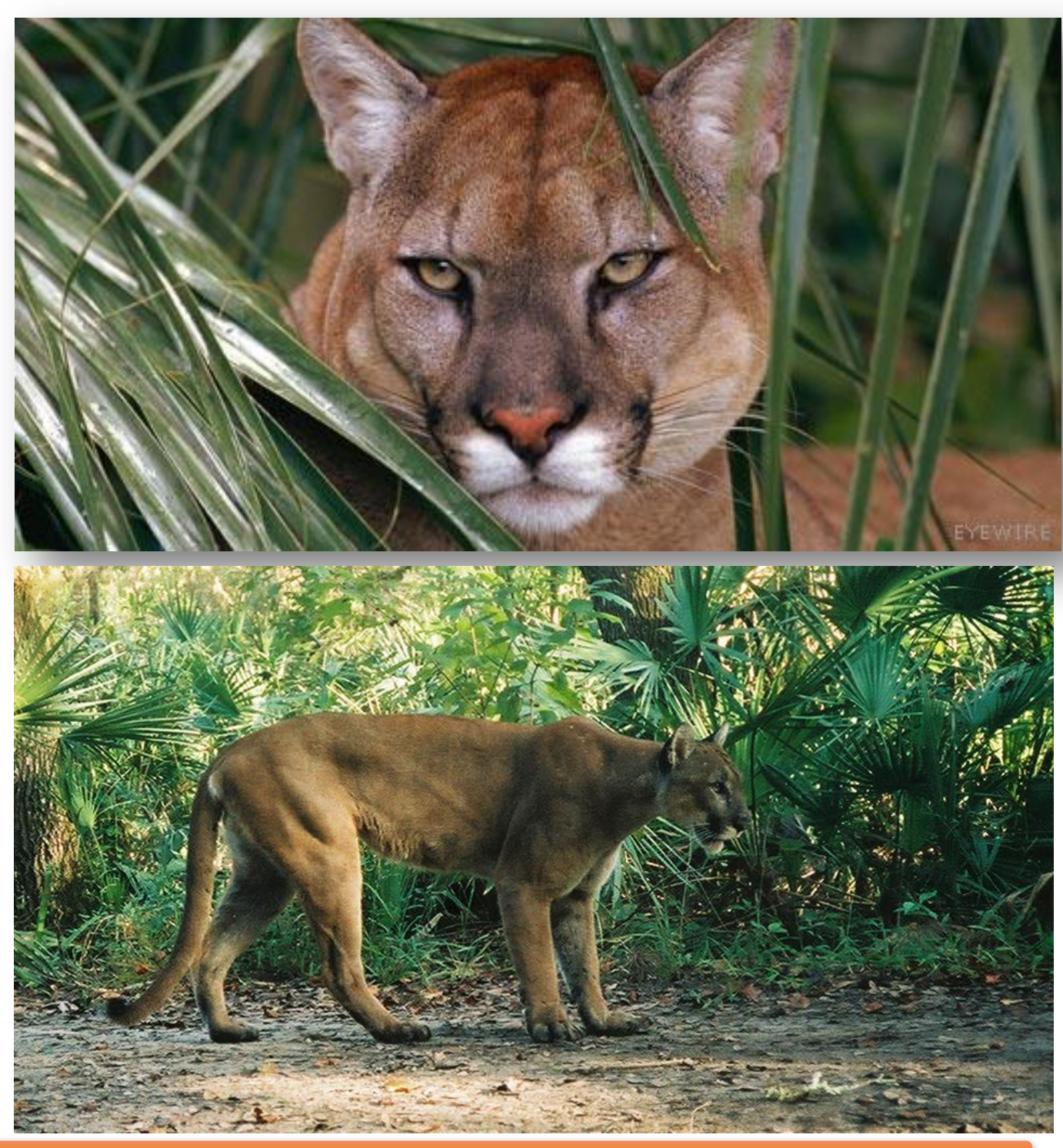




\section{USFWS Pilot Program}

- Eligible lands:

- Primary and dispersal zones of the Panther Focus Area

- Parcels $\geq 50$ acres

- Payments:

- Tier 1 lands: \$30.80/acre for burning, mechanical vegetation treatment, invasive control

- 190,541 eligible acres

- Tier 2 lands: \$9/acre for prescribed grazing plan

- 69,194 eligible acres

- 5 year contract

- Safe harbor agreement in the expansion area

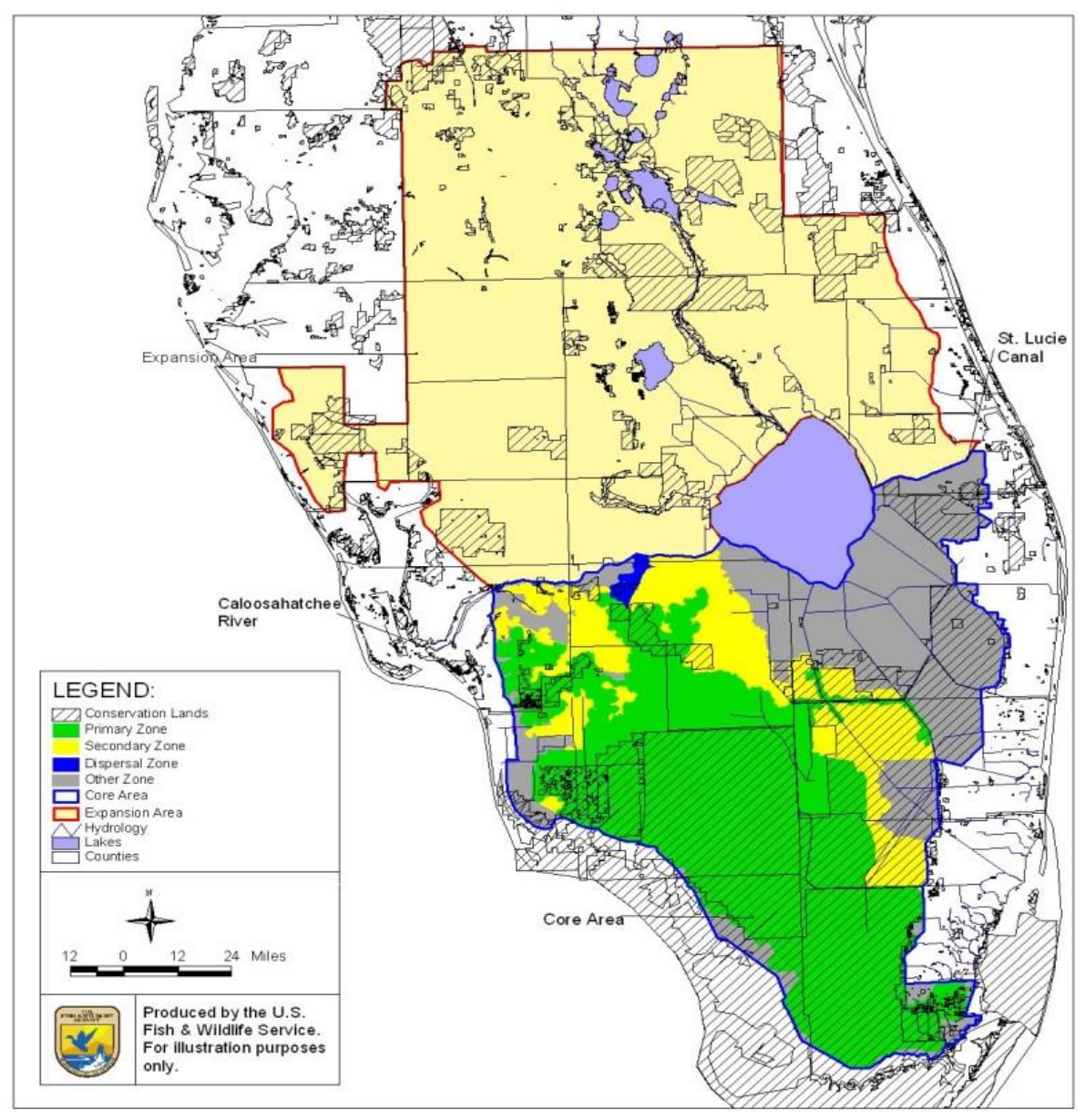




\section{Objectives}

Delisting of the Florida panther necessitates that habitat be conserved on private range lands ${ }^{1}$.

Objectives:

- Identify cattlemen preferences for panther/habitat conservation programs.

- Determine the size of incentives needed to engage cattlemen in habitat conservation.

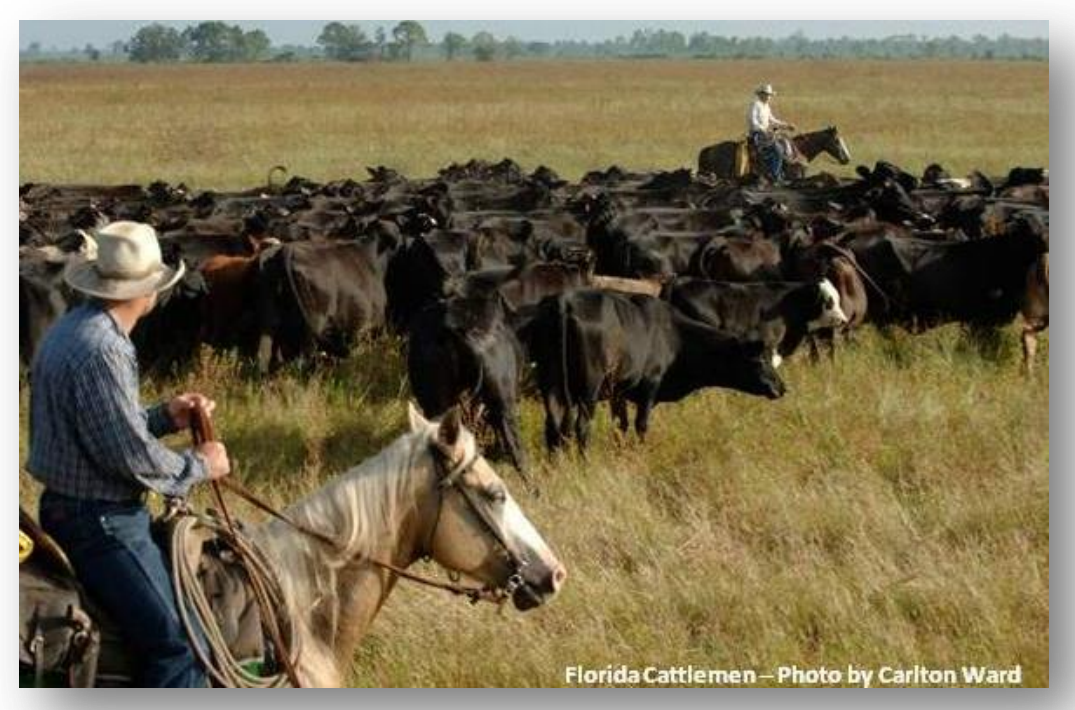

${ }^{1}$ Florida Panther Recovery Plan (Puma concolor coryi), Third Revision. U.S. Fish and Wildlife Service. Atlanta, Georgia. 217 pp. 


\section{Conserving the Panther on Private Rangelands}

- Florida cattle ranches:

- low-intensity land use operations

- mosaic of habitat types that benefit the panther and its prey base

- land stewardship practices

- control of invasive species; prescribed fire; livestock grazing, managing for game species

- Cattlemen's role in panther conservation:

- Cultural stressors

- Economic stressors

- Cattlemen-panther conflicts

- Societal conflicts

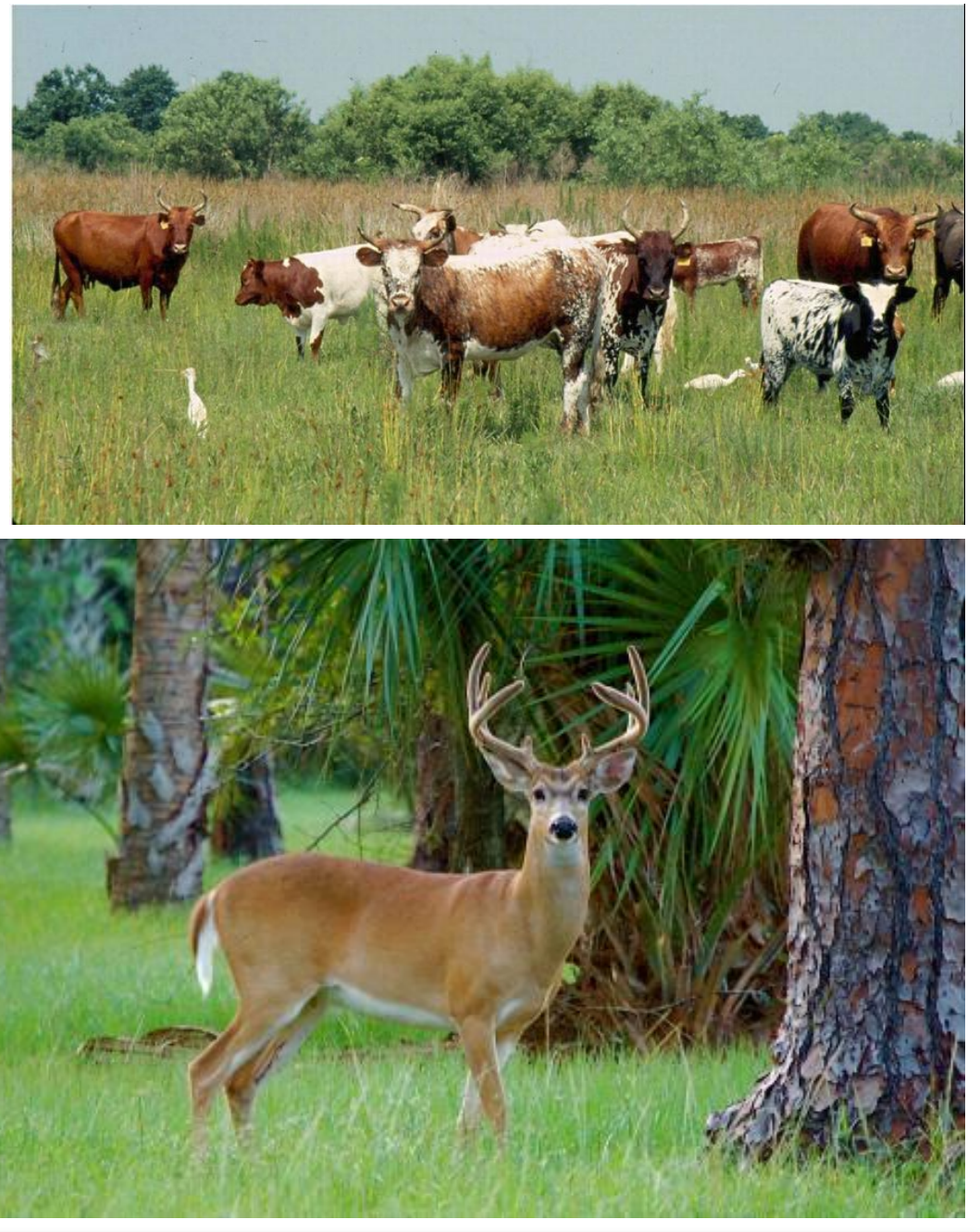




\section{Survey Response and Respondent Characteristics}

- Returned Surveys: 267

- Completed Surveys: 192 (72\%)

- $85.7 \%$ Male

- $96.2 \%$ White

- $83.4 \% 50+$ years in age

- $84.0 \%$ Some college

- $45.0 \%$ Income less than $\$ 100,000$

- $50.4 \%$ Less than $25 \%$ income from beef

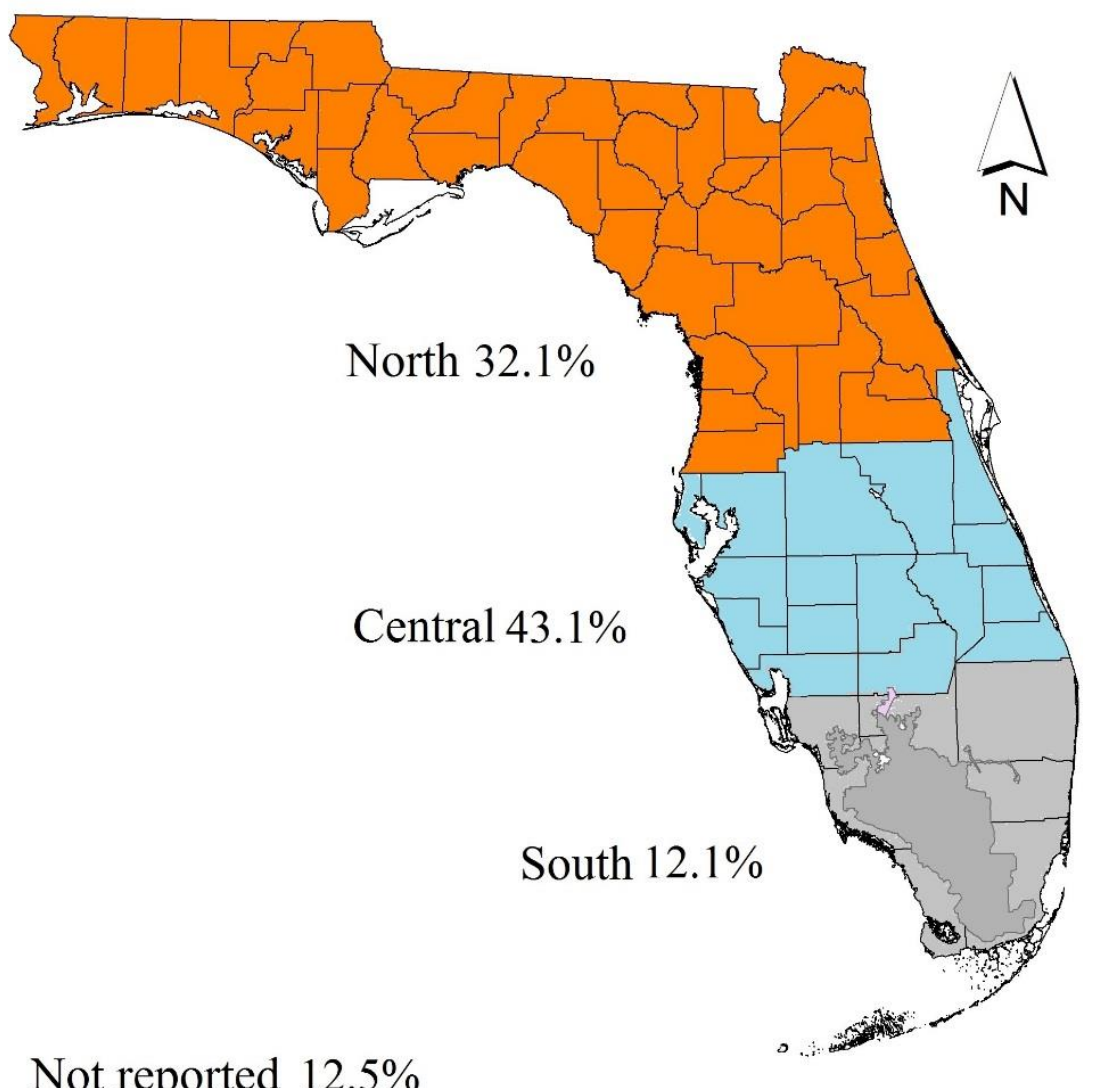




\section{Ranch Operations}

Land Ownership:

- $80 \%$ own land

- $53 \%$ own less than 500 acres

- $10 \%$ own over 5000 acres

- $40 \%$ lease land

- $30 \%$ lease less than 500 acres

Livestock:

- $65 \%$ have less than 250 head of cattle

- $6 \%$ reported livestock depredation due to

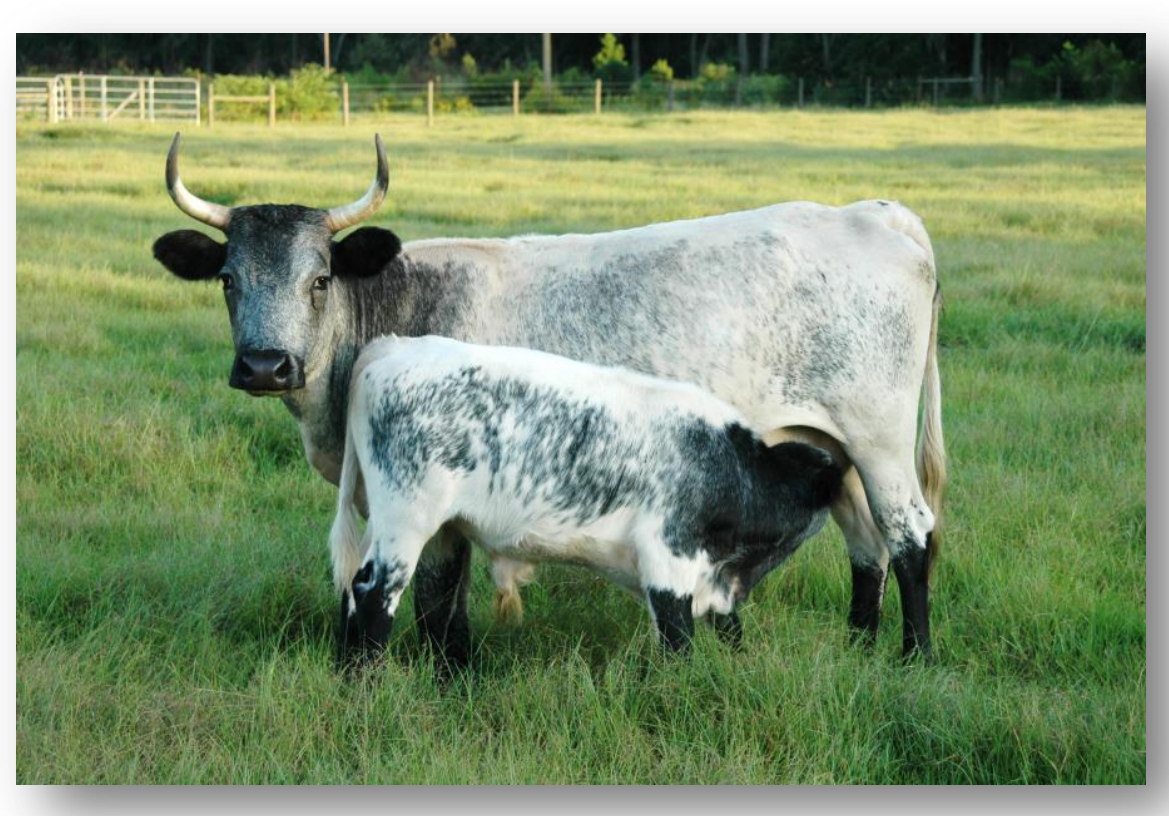
panther 


\section{Attitudes Towards the Panther}

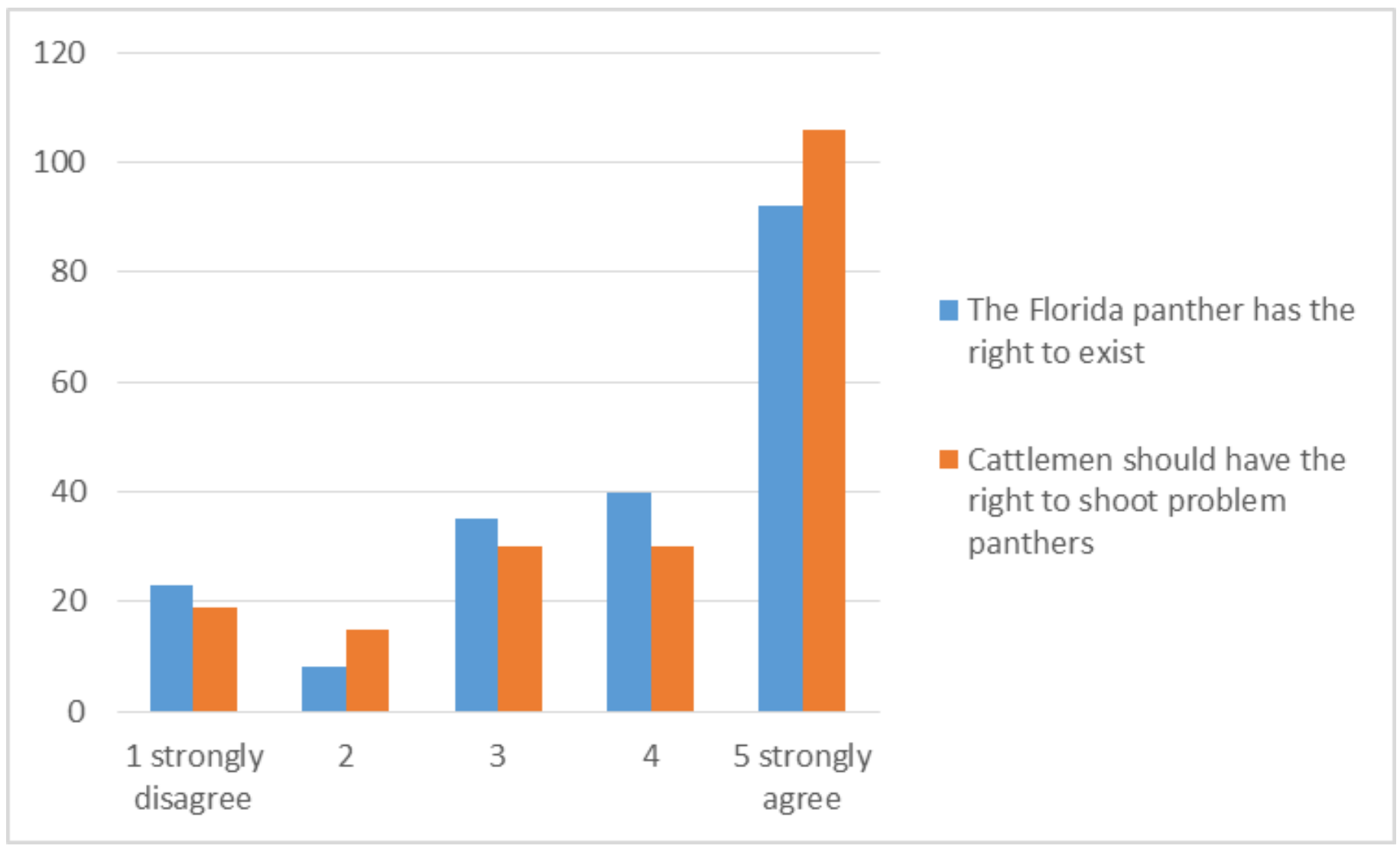

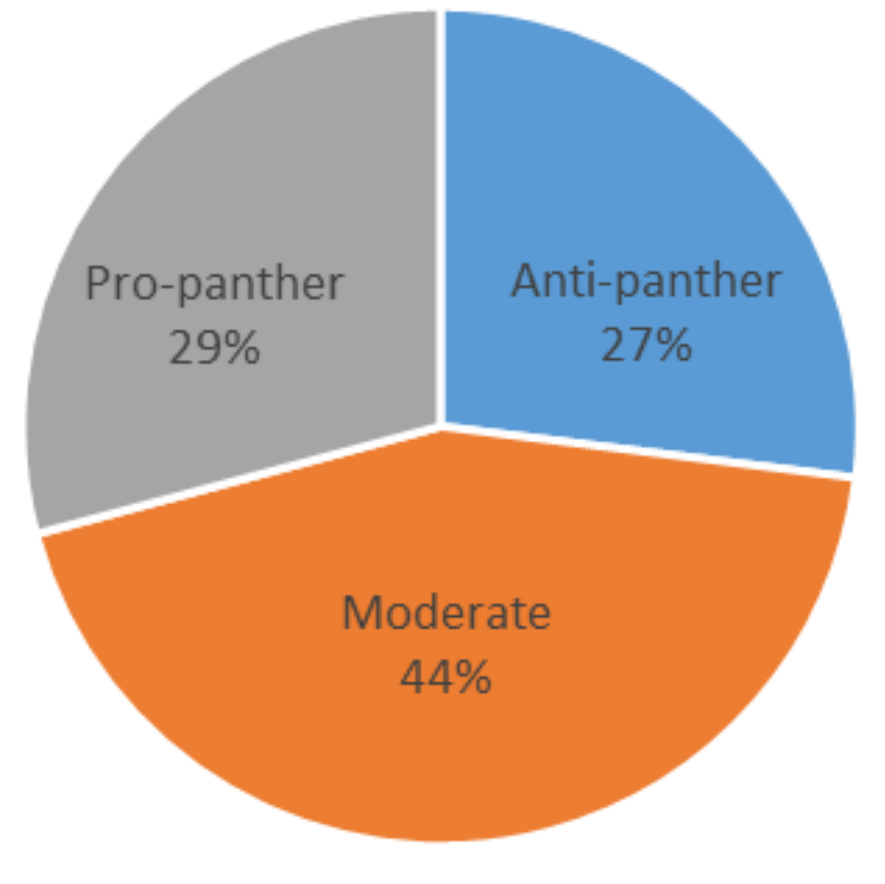

Anti-panther

7-16

Moderate

Pro-panther

17-25

26-35 


\section{Best-Worst Choice Experiments}

- Technical assistance:

- Improved game populations

- Secure water resources

- Improved land stewardship practices

- Identify other stewardship incentives

- Incentive:

- Habitat payment (\$5 - \$30/acre)

- Reduced estate tax (2\% - 5\%)

- Depredation payment ( $1 \%$ - $4 \%$ of annual calf crop)

- Safe harbor agreement
- Monitoring organization:

- USDA - federal

- USFWS - federal

- FWC - state

- Independent environmental organization

- Acres (pasture \& habitat) enrolled:

- $25 \%, 50 \%, 75 \%, 100 \%$

- Contract duration:

- 5, 10, 20, 30 years 


\section{Choice Experiment}

\begin{tabular}{|lcc|}
\hline C3 Habitat Protection Program A & $\begin{array}{c}\text { 1. Prefer Most } \\
\text { (Check only one })\end{array}$ & $\begin{array}{c}\text { 2. Prefer Least } \\
\text { (Check only one })\end{array}$ \\
\hline Annual payment for wildlife habitat: $\$ 10$ acre & $\bigcirc$ & $\bigcirc$ \\
Advice about securing water resources & $\bigcirc$ & $\bigcirc$ \\
Acres enrolled: $75 \%$ of eligible acres & $\bigcirc$ & $\bigcirc$ \\
Time commitment: 5 years & $\bigcirc$ & \\
Monitoring: USDA & $\bigcirc$ & \\
\hline $\begin{array}{l}\text { Would you enroll in Program A exactly as described } \\
\text { above if it was the only program offered? }\end{array}$ & 3. YES $\bigcirc$ NO $\bigcirc$ \\
\hline
\end{tabular}




\section{Attribute Impacts}

\begin{tabular}{|l|c|c|c|c|}
\hline & Coefficient & Standard Error & Z value & P Value \\
\hline $\begin{array}{l}\text { Technical } \\
\text { assistance }\end{array}$ & 2.232 & 0.160 & 13.91 & 0.000 \\
\hline $\begin{array}{l}\text { Incentive type } \\
\text { Acres enrolled }\end{array}$ & 1.917 & 0.170 & 11.24 & 0.000 \\
\hline $\begin{array}{l}\text { Monitoring } \\
\text { agency }\end{array}$ & 0.639 & 0.146 & 10.90 & 0.000 \\
\hline $\begin{array}{l}\text { Contract } \\
\text { duration }\end{array}$ & 0.000 & - & 4.16 & 0.000 \\
\hline
\end{tabular}




\section{Level Scale Values}

\begin{tabular}{|l|c|c|c|c|}
\hline & Coefficient & Standard Error & Z value & P Value \\
\hline Technical assistance: & & & \\
\hline Improve game populations & 1.225 & - & - & - \\
\hline Identify other incentives & 0.082 & 0.134 & 0.61 & 0.541 \\
\hline Improve stewardship & -0.350 & 0.163 & -2.14 & 0.032 \\
\hline Secure water resources & -0.957 & 0.159 & -5.99 & 0.000 \\
\hline Incentive type: & & & & 0.000 \\
\hline Habitat payment & 1.669 & 0.197 & $\mathbf{8 . 4 5}$ & $\mathbf{0 . 0 0 0}$ \\
\hline Reduce estate tax & $\mathbf{0 . 7 4 4}$ & $\mathbf{0 . 1 7 4}$ & $\mathbf{4 . 2 6}$ & $\mathbf{0 . 0 0 3}$ \\
\hline Depredation payment & $\mathbf{0 . 5 1 5}$ & $\mathbf{0 . 1 7 4}$ & $\mathbf{3 . 0 2}$ & - \\
\hline Safe harbor agreement & -2.928 & - & - & \\
\hline
\end{tabular}




\section{Level Scale Values}

\begin{tabular}{|l|c|c|c|c|}
\hline & Coefficient & Standard Error & Z value & P Value \\
\hline Acres enrolled: & & & & 0.000 \\
\hline $\mathbf{1 0 0 \%}$ & 1.078 & 0.165 & 6.53 & - \\
\hline $75 \%$ & -0.253 & - & - & 0.034 \\
\hline $\mathbf{5 0 \%}$ & -0.323 & 0.152 & -2.12 & 0.001 \\
\hline $\mathbf{2 5 \%}$ & -0.502 & 0.149 & -3.36 & 0.000 \\
\hline Contract duration: & & & & 0.000 \\
\hline $\mathbf{5}$ years & 1.475 & 0.177 & 8.31 & 0.038 \\
\hline $\mathbf{1 0}$ years & 0.975 & 0.170 & $\mathbf{5 . 7 2}$ & - \\
\hline $\mathbf{3 0}$ years & -0.315 & 0.152 & -2.07 & - \\
\hline 20 years & -2.135 & - & & 0 \\
\hline
\end{tabular}




\section{Level Scale Values}

\begin{tabular}{|c|c|c|c|c|}
\hline & Coefficient & Standard Error & $Z$ value & P Value \\
\hline \multicolumn{5}{|l|}{ Monitoring agency: } \\
\hline $\begin{array}{l}\text { Florida Fish and Wildlife } \\
\text { Conservation Commission } \\
\text { (FWC) }\end{array}$ & 0.221 & 0.145 & 1.53 & 0.127 \\
\hline $\begin{array}{l}\text { United States Department of } \\
\text { Agriculture (USDA) }\end{array}$ & 0.150 & 0.158 & 0.95 & 0.341 \\
\hline Environmental consultant & -0.004 & - & - & - \\
\hline $\begin{array}{l}\text { United States Fish and } \\
\text { Wildlife Service }\end{array}$ & -0.367 & 0.142 & -2.58 & 0.010 \\
\hline
\end{tabular}




\section{Order of Preferences for Program Attributes}
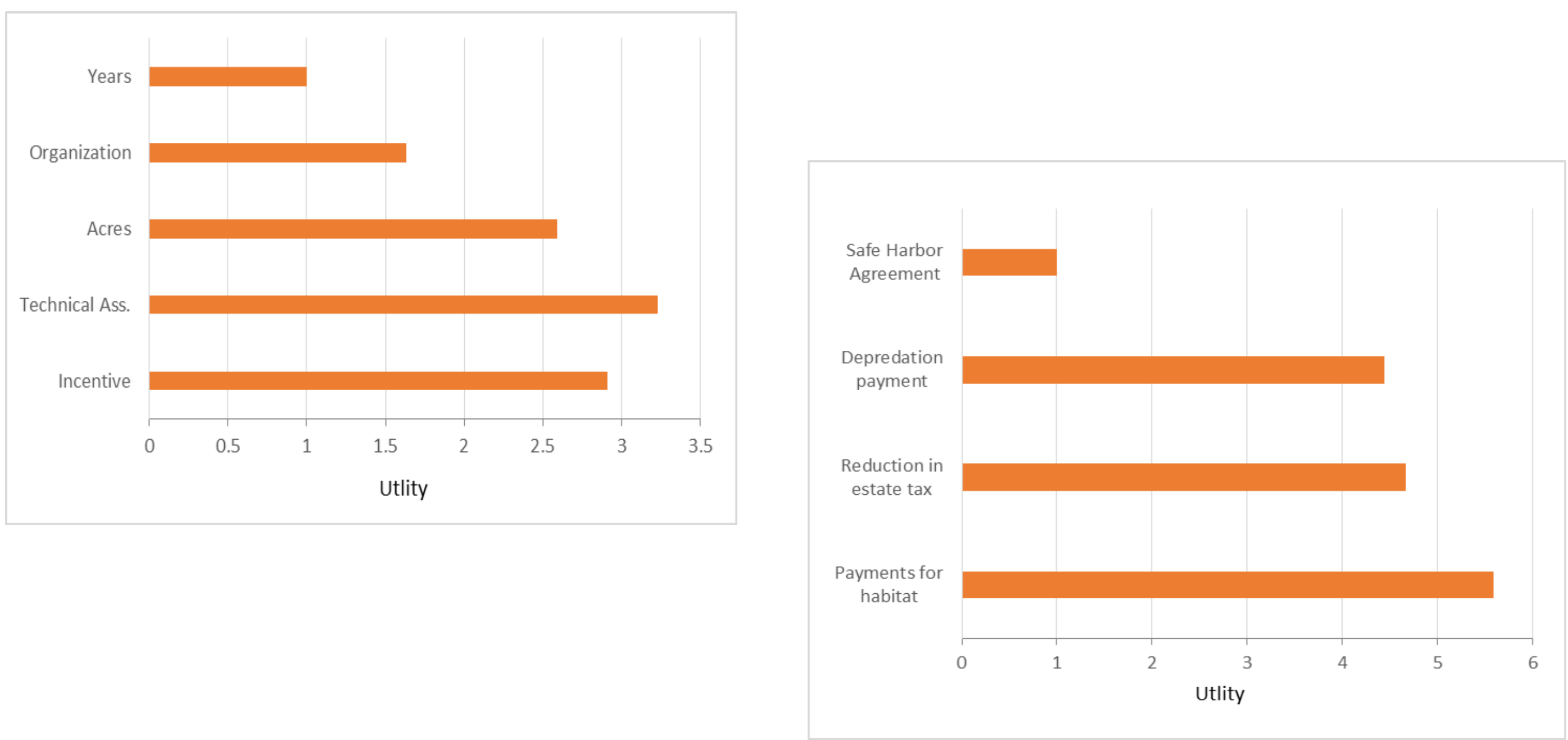


\section{Concluding Comments}

- Low response rate:

- Results may not be representative for Florida cattlemen community

- Should not use findings to project program enrollment

- Utility depends on incentives offered and program features that impact cultural values and personal autonomy

- Most negative impacts with panther $\rightarrow$ focus on monitoring agency and acres enrolled

- Trust in government, private property rights, personal autonomy

- Financial concerns were secondary 


\section{Management Implications}

- Contract obligation (monitoring) and complexity (demonstrate conservation output) affect landowners' willingness to enroll in conservation programs

- Incentives for habitat (not species) conservation may provide cognitive separation from species recovery efforts

- Landowners who are critical of government and species recovery programs

- Avoid collection of unnecessary sensitive information

- Concerns about future regulation and misuse of information by agencies

- Reframe programs in terms of 'fair exchange' in the present

- Use trusted agency to implement program 